\title{
Costos de los tratamientos en úlceras venosas: revisión de literatura 2015-2020
}

\section{Venous ulcer treatment costs: Literature review 2015-2020}

\author{
Leydy J. Rodríguez-Suárez y Nelson R. Campos-Guzmán* \\ Facultad de Ciencias de la Salud, Universidad de Ciencias Aplicadas y Ambientales, Bogotá, Colombia
}

\begin{abstract}
Resumen
Introducción: El tratamiento de las úlceras venosas implica elección fundamentada del tratamiento que disminuya la hipertensión venosa, acelere la cicatrización, por su frecuencia de uso y disminución de costos, existiendo alternativas de tratamiento en el mercado. Objetivo: Describir la relación de costos en los diferentes tratamientos para el manejo de úlceras venosas, reportado en la literatura cientifica. Metodología: Estudio de revisión de literatura estructurada en tres fases, la búsqueda se realizó con las palabras clave: úlceras venosas, tratamiento en úlceras venosas, costos en tratamiento, costos en salud. Criterios de inclusión: artículos publicados entre los años 2015-2020, en inglés, español y portugués. Resultados: Se incluyeron 39 artículos para la descripción de costos. Las úlceras venosas son de gran impacto económico para el sector de la salud, en los países occidentales los costos de estos tratamientos representan el 2-5\% del presupuesto total de salud y actualmente existe variada disponibilidad de tratamientos invasivos y no invasivos con su respectivo impacto económico. Conclusión: Existen diferentes abordajes y dispositivos a nivel mundial para el tratamiento de úlceras venosas y su aplicación depende de la disponibilidad; en la revisión se hallaron costos por tratamientos invasivos y no invasivos, los cuales varían de la inversión de cada país.
\end{abstract}

Palabras clave: Úlceras venosas. Tratamiento en úlceras venosas. Costos en tratamiento. Costos en salud.

\section{Abstract}

Background: the treatment of venous ulcers implies a well-founded choice of treatment that reduces venous hypertension, accelerates healing, due to its frequency of use and cost reduction, there are treatment alternatives on the market. Objective: To describe the cost relationship in the different treatments for the management of venous ulcers, reported in the scientific literature. Methodology: A 3-phase structured literature review study, the search was carried out according to the key words: venous ulcers, treatment of venous ulcers, treatment costs, health costs. Inclusion criteria: articles published between the years 2015-2020, in different languages English, Spanish and Portuguese. Results: 39 articles were included for the description of costs, venous ulcers have a great economic impact for the health sector, in western countries the costs of these treatments represent $2 \%-5 \%$ of the total health budget and currently there is a wide availability of invasive and non-invasive treatments with their respective economic impact. Conclusion: There are different approaches and devices worldwide for the treatment of venous ulcers and their application depends on availability; In the review, costs for invasive and non-invasive treatments were found, which vary from the investment of each country.

Key words: Venous ulcers. Treatment of venous ulcers. Treatment costs. Health costs.

Correspondencia:

*Nelson R. Campos-Guzmán

E-mail: ncampos@udca.edu.co
Fecha de recepción: 12-03-2021

Fecha de aceptación: 07-09-2021

DOI: 10.24875/RMA.21000015
Disponible en internet: 29-11-2021 Rev Mex Angiol. 2021;49(4):123-132 www.RMAngiologia.com 0377-4740/@ 2021 Sociedad Mexicana de Angiología y Cirugía Vascular y Endovascular, A.C. Publicado por Permanyer. Este es un artículo open access bajo la licencia CC BY-NC-ND license (http://creativecommons.org/licenses/by-nc-nd/4.0/). 


\section{Introducción}

La insuficiencia venosa periférica es la incapacidad de las venas para realizar el retorno de la sangre al corazón, provocando la acumulación de este fluido en las piernas, dando lugar a la sintomatología de la insuficiencia venosa crónica (IVC) ${ }^{1}$. Dicho fenómeno se acentúa en el sedentarismo, la hipotensión ortostática y por el efecto de la bipedestación, que obliga al aparato circulatorio a realizar un sobreesfuerzo para conseguir que la sangre retorne al corazón².

Desde la fisiopatología hay evidencia que en la úlcera venosa (UV) se presenta reflujo valvular, aumentando la presión venosa ambulatoria y de esta forma conduciendo a la hipertensión venosa ${ }^{3,4}$, en donde la transmisión de la hipertensión venosa a la microcirculación dérmica causa extravasación de eritrocitos, que son los estímulos para desligar la lesión inflamatoria. La activación de la microcirculación produce la liberación de citocinas y factores de crecimiento que permiten la migración de leucocitos al intersticio, situándose alrededor de los capilares desencadenando la activación de factores de crecimiento, lo que se acoplaría a los fibroblastos, manifestándose en los trastornos dérmicos observados en los estados avanzados de IVC como las UV 5 .

Las úlceras venosas crónicas son una patología frecuente, con una incidencia de 3 a 5 nuevos casos por mil personas al año. Presentan una relación mujer: hombre de 3:1, con una prevalencia del 0.1 al $0.3 \%$ en la población europea, aumentando a $1 \%$ en mayores de 65 años $^{6}$. "Las UVC representan entre un 80 y $90 \%$ de las heridas de miembros inferiores, las cuales pueden ser producidas por enfermedades isquémicas derivadas de la alteración vascular 0 arterial; las alteraciones venosas producen heridas varicosas entre el 70 y $90 \%$, estimando su tratamiento como de alto costo por su cronicidad»?

Las UV requieren periodos para su curación que pueden ir más allá de seis semanas, cicatrizando por segunda intención ${ }^{8}$. Roche $(2017)^{9}$ refiere que las UV pueden ser únicas o múltiples, poco profundas y/o exudativas.

El tratamiento para las UV se adapta al paciente según su grado de complejidad o estadio de la herida, implica una adecuada elección de los apósitos con base en una fundamentación literaria, donde explique el tiempo de cicatrización, su frecuencia de uso y sus costos $^{6}$.

Los costos son una importante herramienta no solo para la toma de decisiones, sino para la elección de alternativas que ayuden a obtener mejores resultados, de esta manera garantizando la mayor eficacia en los servicios prestados logrando el éxito del binomio calidad-eficiencia ${ }^{10,11}$.

Al analizar el núcleo de la enfermedad, se logra diseñar una política adecuada en la distribución de recursos y comparándolos con la relación costo-resultados. De ahí que los costos aplicados en una patología sean necesarios para asignar recursos, realizar costos comparativos; estos costos se consideran importantes, ya que demuestran el impacto económico de la enfermedad en la sociedad considerando la tasa de morbilidad y mortalidad asociadas a la enfermedad, en este caso las UV'10.

El objetivo de este artículo es describir la relación de costos en los diferentes tratamientos para el manejo de UV reportado en la literatura científica.

\section{Metodología}

Estudio de revisión de literatura estructurado en tres fases. Fase uno: búsqueda de información en bases de datos (Science direct, PubMed, Scielo y Clinical Key). Fase dos: se agrupó la información por tipo de tratamiento (apósitos, ablación por radiofrecuencia, escleroterapia con espuma, cirugía y coadyuvantes como UrgoStart $^{\circledR}$, polisacárido de zinc y carboximetilcelulosa) en una ficha analítica resumen en educación (ficha RAE), clasificándolos por tipo de tratamiento. Fase tres: análisis y discusión de los resultados a partir de las temáticas encontradas.

La búsqueda se realizó de acuerdo con las palabras clave: úlceras venosas, tratamiento en úlceras venosas, costos en tratamiento, costos en salud. Criterios de inclusión: artículos publicados entre los años 20152020, en inglés, español y portugués, y tratamiento de venas o UV, estudios de costo beneficio para tratamiento de UV y estudios experimentales. Criterios de exclusión: artículos que incluyeran costos de tratamientos úlceras de etiología arterial, artículos que incluyeran costos de complicaciones derivados del tratamiento y artículos que carecieran de información sobre costos, del total de la búsqueda se lograron obtener 39 artículos con los criterios de inclusión.

\section{Resultados}

Con la ecuación de búsqueda se encontraron 90 artículos, se excluyeron 51 artículos por ventana de publicación, por carencia de información sobre costos y objetivo de tratamiento dirigido a úlceras arteriales.

Actualmente existe variada disponibilidad de tratamientos invasivos y no invasivos, con diferentes 
Tabla 1. Comparación de los tratamientos, países y costos

\begin{tabular}{|c|c|c|c|c|c|}
\hline País & $\begin{array}{l}\text { Datos } \\
\text { sociodemográficos y } \\
\text { descripción de estudios }\end{array}$ & Tratamiento & Costo & Ventajas & Desventajas \\
\hline $\begin{array}{l}\text { Alemania } \\
\text { Noppeney, } \\
\text { et al., } 2016^{12}\end{array}$ & $\begin{array}{l}89,647 \text { pacientes ( } 27,463 \\
\text { hombres, } 62,184 \\
\text { mujeres, edad promedio } \\
52.8 \text { años, rango } 15 ; \text { se } \\
\text { analizaron } 96 \text { años) } \\
\text { recogidos en el registro } \\
\text { de la cirugía de control } \\
\text { de calidad de la } \\
\text { Sociedad Alemana de } \\
\text { Cirugía Vascular entre } \\
2001 \text { y 2009. En estos } \\
\text { pacientes, } 95,214 \\
\text { procedimientos } \\
\text { quirúrgicos se } \\
\text { realizaron en } 105,296 \\
\text { miembros. Los } \\
\text { resultados del estudio } \\
\text { se organizaron según la } \\
\text { clasificación en la tabla } \\
\text { CEAP }\end{array}$ & Cirugía & $\begin{array}{l}\text { USD } 947 \text { mil } \\
\text { millones al año }\end{array}$ & $\begin{array}{l}\text { Abordaje mínimo, } \\
\text { ambulación } \\
\text { precoz, no } \\
\text { requieren } \\
\text { internamiento, } \\
\text { reinserción rápida } \\
\text { a su actividad } \\
\text { laboral y resultado } \\
\text { cosmético } \\
\text { excelente }^{11}\end{array}$ & $\begin{array}{l}\text { Sangrado, } \\
\text { infección, lesión } \\
\text { vascular y } \\
\text { neurológica, } \\
\text { trombosis venosa } \\
\text { profunda, } \\
\text { hematomas, } \\
\text { tromboflebitis } \\
\text { superficial, y una } \\
\text { alta incidencia de } \\
\text { recurrentes a los } \\
5 \text { años } \\
\text { La reincidencia a } \\
\text { los } 2 \text { años es del } \\
20-50 \% \text { y a los } 10 \\
\text { años es del } 70 \%\end{array}$ \\
\hline $\begin{array}{l}\text { Irlanda } \\
\text { Elrasheid, } \\
\text { et al., 2016 }\end{array}$ & $\begin{array}{l}\text { Revisión sistemática y } \\
\text { metaanálisis, } 15 \\
\text { estudios se incluyeron } \\
\text { en la revisión. } 10 \\
\text { artículos informaron los } \\
\text { resultados de nueve } \\
\text { ensayos controlados } \\
\text { aleatorizados } \\
3 \text { artículos de los } \\
\text { ensayos aleatorizados y } \\
2 \text { informes de estudios } \\
\text { de cohortes } \\
\text { retrospectivos. Para la } \\
\text { cirugía abierta, úlcera } \\
\text { curación es } \\
\text { significativamente mejor } \\
\text { en comparación con la } \\
\text { compresión, único } \\
\text { grupo cuando se } \\
\text { analizaron los datos } \\
\text { agrupados (78.8 vs. } \\
71.3 \% \text { ) o datos de } \\
\text { ensayo aleatorizado } \\
\text { controlado (79 vs. } \\
73.4 \% \text { ). Riesgo relativo } \\
\text { de } 1.00 \text { [1.01-1.16]; IC del } \\
95 \%, P=0.03 \text { para todos } \\
\text { los estudios }\end{array}$ & $\begin{array}{l}\text { Cirugía vs. } \\
\text { compresión }\end{array}$ & & $\begin{array}{l}\text { Mejora las tasas } \\
\text { de } \\
\text { recurrencia<tasa } \\
\text { de curación de las } \\
\text { úlceras }\end{array}$ & \\
\hline $\begin{array}{l}\text { Irlanda } \\
\text { Aherne, et al., } \\
2014^{14}\end{array}$ & $\begin{array}{l}296 \text { pacientes fueron } \\
\text { sometidos a tratamiento } \\
\text { quirúrgico, } 66 \% \text { de los } \\
\text { pacientes eran mujeres. } \\
\text { Un total de } 204 \\
\text { pacientes tuvieron } \\
\text { ablación por } \\
\text { radiofrecuencia. La } \\
\text { edad media de los } \\
\text { pacientes fue de } \\
49.1 \pm 1.3\end{array}$ & $\begin{array}{l}\text { Ablación con } \\
\text { radiofrecuencia vs. } \\
\text { cirugía }\end{array}$ & USD 198.47 & $\begin{array}{l}\text { Aumenta calidad } \\
\text { de vida del } \\
\text { paciente }\end{array}$ & $\begin{array}{l}\text { Reducción del } \\
\text { dolor, parestesia } \\
\text { postoperatoria }\end{array}$ \\
\hline
\end{tabular}


Tabla 1. Comparación de los tratamientos, países y costos (continuación)

\begin{tabular}{|c|c|c|c|c|c|}
\hline País & $\begin{array}{l}\text { Datos } \\
\text { sociodemográficos y } \\
\text { descripción de estudios }\end{array}$ & Tratamiento & Costo & Ventajas & Desventajas \\
\hline & $\begin{array}{l}\text { De los pacientes } \\
\text { sometidos a ablación por } \\
\text { radiofrecuencia, } 160 \\
\text { pacientes }(78 \%) \text { se } \\
\text { realizaron como cirugía } \\
\text { ambulatoria en } \\
\text { comparación con } 17(18 \%) \\
\text { de los que se someten a } \\
\text { ligadura abierta y } \\
\text { reextracción }(p=0,001)\end{array}$ & & & & \\
\hline $\begin{array}{l}\text { Irán } \\
\text { Alireza, et al., } \\
2019^{15}\end{array}$ & $\begin{array}{l}\text { Comparación de la } \\
\text { escleroterapia con } \\
\text { espuma frente a la } \\
\text { ablación por } \\
\text { radiofrecuencia estudio } \\
\text { simple ciego paralelo } \\
\text { ensayo clínico } \\
\text { aleatorizado } \\
60 \text { pacientes de edad } \\
18-75 \text { años con venas } \\
\text { varicosas primarias } \\
\text { debido a la } \\
\text { incompetencia }\end{array}$ & $\begin{array}{l}\text { Ablación por } \\
\text { radiofrecuencia vs. } \\
\text { escleroterapia con } \\
\text { espuma }\end{array}$ & $\begin{array}{l}\text { Costo de la } \\
\text { ablación por } \\
\text { radiofrecuencia } \\
\text { más costosa que la } \\
\text { escleroterapia con } \\
\text { espuma. Media de } \\
\text { los costes (costes } \\
\text { de intervención y } \\
\text { hospitalización) sin } \\
\text { cobertura de } \\
\text { seguro en grupos } \\
\text { ablación por } \\
\text { radiofrecuencia y la } \\
\text { escleroterapia con } \\
\text { espuma fueron } \\
3.05 \text { (0.0068) y } \\
0.8(0.0074) \text { millones } \\
\text { Tomans ( }<<0.0001) ; \\
1 \text { US\$ }=11.000 \\
\text { Tomans }\end{array}$ & $\begin{array}{l}\text { Menos invasiva, } \\
\text { reducción de } \\
\text { morbilidad } \\
\text { perioperatoria, } \\
\text { reducción del } \\
\text { tiempo de } \\
\text { recuperación, } \\
\text { reincidencia en } \\
8.5 \% \text { por sus } \\
\text { complicaciones } \\
\text { Más rentable }\end{array}$ & \\
\hline $\begin{array}{l}\text { Italia } \\
\text { Critello, et al., } \\
2019^{16}\end{array}$ & $\begin{array}{l}\text { La escleroterapia con } \\
\text { espuma es un } \\
\text { procedimiento con } \\
\text { invasividad reducida } \\
\text { que implica el } \\
\text { tratamiento local de } \\
\text { dilataciones venosas } \\
\text { como las venas } \\
\text { varicosas }\end{array}$ & $\begin{array}{l}\text { Escleroterapia con } \\
\text { espuma }\end{array}$ & & & $\begin{array}{l}\text { Eventos adversos } \\
\text { neurológicos } \\
\text { graves, burbujas } \\
\text { persistentes en la } \\
\text { circulación } \\
\text { sistémica que } \\
\text { pueden causar el } \\
\text { bloqueo temporal } \\
\text { de los vasos }\end{array}$ \\
\hline $\begin{array}{l}\text { Colombia } \\
\text { Nova, et al., } \\
2017^{17}\end{array}$ & $\begin{array}{l}\text { Los resultados muestran } \\
\text { que los costos directos } \\
\text { del tratamiento de la } \\
\text { curación avanzada } \\
\text { durante cinco meses son } \\
\text { menores que los costos } \\
\text { generados durante los } 48 \\
\text { meses de tratamiento } \\
\text { con curación } \\
\text { convencional. Así mismo, } \\
\text { se puede observar que la } \\
\text { curación avanzada puede } \\
\text { ser más costosa } \\
\text { inicialmente por el } \\
\text { aumento derivado por los } \\
\text { costos tanto en } \\
\text { suministros como del } \\
\text { profesional especializado, } \\
\text { pero estos solo } \\
\text { representan el } 16.5 \%\end{array}$ & Apósitos & USD $1,000-6,000$ & $\begin{array}{l}\text { Aumenta la } \\
\text { capacidad de } \\
\text { autolisis, } \\
\text { disminuyendo la } \\
\text { infección y el } \\
\text { dolor sobre la } \\
\text { herida, tasa de } \\
\text { reincidencia } \\
\text { menor al 15\% }\end{array}$ & $\begin{array}{l}\text { Tamaño mayor al } \\
40 \% \text {, tejido } \\
\text { fibroso }>50 \%\end{array}$ \\
\hline
\end{tabular}


Tabla 1. Comparación de los tratamientos, países y costos (continuación)

\begin{tabular}{|c|c|c|c|c|c|}
\hline País & $\begin{array}{l}\text { Datos } \\
\text { sociodemográficos y } \\
\text { descripción de estudios }\end{array}$ & Tratamiento & Costo & Ventajas & Desventajas \\
\hline $\begin{array}{l}\text { Reino Unido } \\
\text { Julian, et al., } \\
2018^{18}\end{array}$ & $\begin{array}{l}\text { Un modelo de decisión se } \\
\text { construyó en TreeAge } \\
\text { Pro (Williamstown, MA, } \\
\text { EE.UU.) que representa la } \\
\text { gestión de las úlceras } \\
\text { venosas de la pierna no } \\
\text { cicatrizan con la atención } \\
\text { estándar o con un apósito } \\
\text { más terapia de } \\
\text { compresión, seguido de la } \\
\text { atención estándar durante } \\
\text { un periodo de } 6 \text { meses } \\
\text { Se esperaba que la } \\
\text { probabilidad de curación } \\
\text { entre las úlceras venosas } \\
\text { de la pierna de } \\
\text { duración>6 meses } 0.49 \text { a } \\
\text { los } 6 \text { meses después del } \\
\text { tratamiento inicial con } \\
\text { una terapia de vestir que } \\
\text { contiene colágeno más } \\
\text { compresión seguido de la } \\
\text { atención estándar en } \\
\text { comparación con } 0.11 \\
\text { entre los pacientes } \\
\text { administrados con la } \\
\text { atención estándar }\end{array}$ & $\begin{array}{l}\text { Apósitos a base de } \\
\text { colágeno vs. } \\
\text { atención estándar }\end{array}$ & $\begin{array}{l}\text { USD } 185.98 \text { de } 7 \text { a } \\
12 \text { meses de } \\
\text { tratamiento }\end{array}$ & $\begin{array}{l}\text { Facilita la } \\
\text { absorción del } \\
\text { exudado } \\
\text { previniendo el } \\
\text { aumento del } \\
\text { tamaño de la } \\
\text { herida e } \\
\text { inhibiendo el } \\
\text { crecimiento de } \\
\text { microorganismos }\end{array}$ & \\
\hline $\begin{array}{l}\text { Reino Unido } \\
\text { Julian, et al., } \\
2017^{19}\end{array}$ & $\begin{array}{l}505 \text { pacientes mayores } \\
\text { de edad con diagnóstico } \\
\text { de úlceras venosas } \\
\text { desde } 2012 \text {, con menos } \\
\text { de } 12 \text { meses de clínica }\end{array}$ & Apósitos & $\begin{array}{l}\text { El coste medio del } \\
\text { Servicio Nacional de } \\
\text { Salud de Reino Unido } \\
\text { de cuidado de } \\
\text { heridas en la } \\
\text { práctica clínica } \\
\text { durante } 12 \text { meses era } \\
\text { un estimado de USD } \\
8,965 \text { por úlcera } \\
\text { venosa en pierna. Sin } \\
\text { embargo, el coste de } \\
\text { la gestión de una } \\
\text { úlcera venosa no } \\
\text { curada fue de } 4 \text { a } 5 \\
\text { veces más que el de } \\
\text { la gestión de una } \\
\text { úlcera venosa } \\
\text { curada (USD } 3,516 \\
\text { por úlcera venosa } \\
\text { curada vs. USD } \\
\text { 15,843 por úlcera } \\
\text { venosa sin cicatrizar) }\end{array}$ & & $\begin{array}{l}\text { Uso de } \\
\text { analgésicos y } \\
\text { antibióticos } \\
\text { durante el } \\
\text { tratamiento }\end{array}$ \\
\hline $\begin{array}{l}\text { Brasil } \\
\text { Januário, et al., } \\
2016^{20}\end{array}$ & $\begin{array}{l}\text { Estudio analítico, } \\
\text { preexperimental. Se } \\
\text { incluyeron } 30 \text { pacientes } \\
\text { con úlceras venosas } \\
\text { refractarias y se les } \\
\text { aplicó vendajes con } \\
\text { carboximetilcelulosa al } \\
20 \% \text { durante } 20 \text { semanas. } \\
\text { El análisis se basó en la } \\
\text { medición del área de } \\
\text { úlceras, realizada en la } \\
\text { primera visita y después } \\
\text { del final del tratamiento }\end{array}$ & $\begin{array}{l}\text { Carboximetilcelulosa } \\
\text { gel } 20 \%\end{array}$ & $\begin{array}{l}\text { USD } 84.92 \text { a USD } \\
187.49 \text { por paciente } \\
\text { en un tiempo de } 20 \\
\text { semanas }\end{array}$ & $\begin{array}{l}\text { Vida útil de } 90 \text { días } \\
\text { Tratamiento de } 20 \\
\text { semanas en la } \\
\text { mayoría de } \\
\text { pacientes }\end{array}$ & $\begin{array}{l}\text { Dolor presente en } \\
\text { el } 80 \% \text { de los } \\
\text { casos }\end{array}$ \\
\hline
\end{tabular}


Tabla 1. Comparación de los tratamientos, países y costos (continuación)

\begin{tabular}{|c|c|c|c|c|c|}
\hline País & $\begin{array}{l}\text { Datos } \\
\text { sociodemográficos y } \\
\text { descripción de estudios }\end{array}$ & Tratamiento & Costo & Ventajas & Desventajas \\
\hline & $\begin{array}{l}\text { Hubo una reducción de } \\
3.9 \mathrm{~cm}^{2} \text { del área de la } \\
\text { lesión ( } \mathrm{p}=0.0001) \text {, } \\
\text { correspondiente al } \\
38.8 \%(\mathrm{p}=0.0001) \text {. No } \\
\text { hubo interrupción del } \\
\text { tratamiento ni aumento } \\
\text { del área de la lesión en } \\
\text { ningún paciente }\end{array}$ & & & & \\
\hline $\begin{array}{l}\text { México } \\
\text { Moreno-Eutimio, } \\
\text { et al., 2016 } 21\end{array}$ & $\begin{array}{l}\text { Estudio clínico } \\
\text { controlado aleatorizado, } \\
\text { comparativo, en } \\
\text { pacientes con úlceras } \\
\text { venosas crónicas para } \\
\text { evaluar el efecto en la } \\
\text { reducción del área de la } \\
\text { úlcera antes y después } \\
\text { de ocho semanas de } \\
\text { tratamiento de } \\
\text { compresión más } \\
\text { aplicación tópica de } \\
\text { polímero polisacárido } \\
\text { con óxido de zinc y se } \\
\text { comparó con pacientes } \\
\text { tratados únicamente } \\
\text { con compresión y } \\
\text { aplicación de apósitos } \\
\text { simples. El tratamiento } \\
\text { tópico con polímero } \\
\text { polisacárido con óxido } \\
\text { de zinc más el sistema } \\
\text { de compresión mostró } \\
\text { una menor área } \\
\text { promedio de la úlcera } \\
\text { con respecto al grupo } \\
\text { control después de } \\
\text { ocho semanas de } \\
\text { seguimiento (p=0.0377), } \\
\text { además de un mayor } \\
\text { porcentaje de reducción } \\
\text { del área de la } \\
\text { úlcera ( } p=0.0189 \text { ) con } \\
\text { respecto al grupo } \\
\text { control }\end{array}$ & $\begin{array}{l}\text { Polímero } \\
\text { polisacárido de } \\
\text { óxido de zinc }\end{array}$ & & $\begin{array}{l}\text { Disminución de las } \\
\text { úlceras venosas } \\
\text { crónicas mayores } \\
\text { a } 20 \mathrm{~cm} \text { en un } 63 \% \\
\text { en } 8 \text { semanas }\end{array}$ & \\
\hline
\end{tabular}

CEAP: Clinical-Etiological-Anatomical-Pathophysiological.

alternativas de economía resumidos en la tabla 1. Para el abordaje del tratamiento de las UV se debe partir de que los tratamientos tienen objetivos específicos como apropiado control de la infección, preparación del lecho de la herida y el proceso de curación, con propiedades como hidratación, aislamiento térmico, eliminación de tejido necrótico, control bacteriano y un $\mathrm{pH}$ adecuado 4 .

La compresión de vendaje multicapa es el gold standard en el manejo de las UV, técnica que logra una curación entre el 68 y el $83 \%$ en 24 semanas, aunque con altas tasas de recurrencia ${ }^{13}$. La cirugía es uno de los métodos populares desde hace 100 años para el tratamiento de las UV, requiriendo un periodo de recuperación total que puede variar dependiendo de las complicaciones, como sangrado, infección, lesión vascular y neurológica, trombosis venosa profunda, tromboflebitis superficial y una alta incidencia de recurrencia a 5 años ${ }^{22}$, además de hematomas, molestias posprocedimiento, flebitis e infección de la piel, dadas las condiciones descritas es un procedimiento invasivo que incrementa costos. 
Dentro de las técnicas mínimamente invasivas se encuentran: ablación térmica endovenosa (ablación con láser, ablación con radiofrecuencia y esclerosis venosa por vapor) y ablación química endovenosa (escleroterapia líquida y de espuma, ablación mecano-química y embolización de cianoacrilato) ${ }^{16}$.

En Europa se encontraron publicaciones de diferentes países para la temática de esta revisión, entre estos está Hungría, en donde las UV representan una carga importante para el sistema de salud, en el 2016 la administración de caja nacional de salud invirtió USD 3.89 millones; que incluyen cuidados agudos para pacientes hospitalizados $44.7 \%$, atención ambulatoria $33 \%$ y productos farmacéuticos $14.1 \%$, en este caso siendo las mujeres un número importante de la población afectada entre el 64 y el $67 \%{ }^{17}$.

En Reino Unido el costo de estos tratamientos está entre USD 1,983 y USD 2,380 por paciente ${ }^{23}$. El Servicio Nacional de Salud de Reino Unido invierte en servicios de la comunidad una cantidad considerable de dinero para el tratamiento de UV, ese costo se estima en alrededor de USD 256 mil millones ${ }^{24}$.

En Alemania los costos anuales para el tratamiento de UV mediante la cirugía se han estimado en USD 9,000 por paciente, estos costos incluyen costos de hospitalización, costos de enfermería, material de vendaje y el tiempo de curación ${ }^{25}$, además se estima que en Alemania se realizan 350,000 procedimientos quirúrgicos cada año, siendo el décimo procedimiento más frecuente, con un costo de 947 millones de dólares al año ${ }^{12}$.

En Irlanda el costo de la ablación por radiofrecuencia oscila entre USD 1,894 y USD 2,130, estos costos incluyen noche en hospitalización, anestesia, sedación intravenosa, laboratorios, ecografía y kit de quirúrgicos; este tratamiento puede tener un costo relativamente alto, varios estudios sugieren esta técnica, ya que el tiempo de intervención disminuye, son procedimientos preferidos ya que reducen el dolor, retorno rápido a la función completa y esto mejora la calidad de vida del paciente, logrando obtener una tasa de éxito del $98 \%$ a las seis semanas de seguimiento ${ }^{14}$.

Una de las alternativas menos invasiva es la escleroterapia con espuma, esta se ha convertido en un procedimiento válido y mínimamente invasivo y rentable; su rentabilidad es evidente a mediano plazo (seis meses) y su tasa de recurrencia es más alta a los cinco años, este tratamiento se puede realizar ambulatoriamente al ser menos agresivo, menos doloroso y sin necesidad de anestesia ${ }^{16}$.
La terapia con apósitos de cura en ambiente húmedo se muestra como una medida terapéutica efectiva y de bajo costo. Gracias al doctor Winter en 1962 se probaron científicamente los beneficios que un medio húmedo tiene sobre el proceso de cicatrización de las heridas ${ }^{17}$, el uso de estos apósitos ocupan entre el 27$22 \%$ del costo total para curar la úlcera ${ }^{3}$.

Existe variedad entre los apósitos, como lo es a base de colágeno tienen un valor de USD 185.98 mensuales por paciente de 7 a 12 meses de tratamiento ${ }^{15}$. El apósito de colágeno bovino fetal ha sido aprobado como un dispositivo clase II para el tratamiento de UV y heridas crónicas, a excepción de las quemaduras de tercer grado; es un apósito de colágeno acelular derivado de animales que se ha procesado y tratado para eliminar elementos celulares, lípidos, carbohidratos y proteínas no colagenosas ${ }^{26}$.

En Reino Unido los costos de los apósitos representan el $15 \%$ de los costos generales del manejo de pacientes con heridas crónicas; los principales factores de costos son las visitas de profesionales de la salud y los ingresos hospitalarios, mientras que los gastos en apósitos llega a USD 242 millones en este país, en donde aproximadamente por paciente tiene un costo de USD 7,187 a USD 2,223 total durante las 40 semanas, en donde se evidenció la curación completa de las úlceras ${ }^{27}$.

En Alemania se encuentran UrgoStart ${ }^{\circledR}$, es un apósito hidroactivo con un costo de USD 557.51, utilizado en el tratamiento hidroactivo de heridas exudativas crónicas como las UV, el tiempo de observación de este tratamiento fue de ocho semanas, evidenciando la curación y mejoría parcial de las heridas; para una completa curación se necesitan entre 20-24 semanas de tratamiento, se considera el éxito de este tratamiento con la reducción de al menos el $40 \%$ de la herida en ocho semanas ${ }^{25}$.

En Brasil se pueden obtener paquetes de apósitos para uso en el domicilio que contiene implementos para las curaciones en el hogar, además de agregar el costo de visita semanal del personal de enfermería valorado en USD 27.64/hora por el concejo federal de enfermería (COFEN), en este estudio se evidenció que el costo total en el hogar fue de USD 11.82 dólares y en clínica de USD 188.75 dólares debido a las 12 consultas de enfermería realizadas para el tratamiento a comparación de los 72 días de los pacientes domiciliarios ${ }^{23}$.

En Brasil se realizó una prueba con carboximetilcelulosa gel $2 \%$, el cual está compuesto por carboximetilcelulosa (ingrediente activo), propilenglicol (agente humectante), metilparabeno (conservante) y 
agua (vehículo), dándole una vida útil de 90 días a este gel, el valor de cada uno de estos tubos por $100 \mathrm{mg}$ fue de USD 3.35 dólares $^{20}$. Es un polímero aniónico derivado de la celulosa, con cualidades de los polisacáridos: solubilidad, viscosidad y capacidad de formar geles. El costo del tratamiento considerando todos los insumos varía entre USD 84.92 y USD 187.49 por paciente en un tiempo de 20 semanas como máximo y la gravedad de la úlcera evidenciando una reducción absoluta en el área de la lesión, en donde el tiempo de duración del tratamiento era de 20 semanas en la mayoría de pacientes. El dolor fue uno de los principales datos clínicos, presente en el $80 \%$ de los casos $^{20}$.

Pycnogenol ${ }^{\circledR}$ es un extracto de la corteza del pino marítimo francés que contiene procianidinas (compuesto químico del Pycnogenol ${ }^{\circledR}$ ) con significativa actividad antioxidante y antiinflamatoria, su administración es por vía oral o tópica y se ha demostrado que tiene efectividad frente al uso de la diosmina/hisperidina para el mismo tratamiento aplicando el uso de apósitos. En los pacientes con tratamiento de Pycnogenol ${ }^{\circledR}$ se evidencia una disminución significativa después de 45 días de tratamiento, evidenciando una cicatrización más favorable; los pacientes con diosmina/hesperidina evidencian mejora después de 60 días; estos tratamientos son utilizados como adyuvantes que disminuyen significativamente el edema de la extremidad afectada $^{28}$.

Las UV en EE.UU. representan entre el 80 y $90 \%$ del total de las heridas de miembros inferiores, su tratamiento ha sido estimado de alto costo debido a la cronicidad de la enfermedad, el $50 \%$ de los pacientes con UV tardaron más de nueve meses para su recuperación y el $20 \%$ dos años más para su recuperación dependiendo del uso de compresión, además el $60 \%$ experimentaron recurrencia en las UV en este país ${ }^{4}$, el costo financiero anual para el tratamiento de heridas crónicas se estima en USD 15 mil millones ${ }^{28}$.

En Irán la ablación por radiofrecuencia se ha convertido en la alternativa menos invasiva, se asocia a una reducción en la morbilidad perioperatoria, el tiempo de recuperación y un aumento en la calidad de vida ${ }^{14}$.

En un metaanálisis realizado en China que incluyó estudios controlados aleatorizados se revisaron diferentes formas de intervenciones con el injerto de piel, entre las cuales se encuentran: autoinjerto (injertos de pellizco e injertos de espesor parcial), autoinjerto y xenoinjerto (injertos de espesor total), aloinjerto y xenoinjerto (queratinocitos cultivados, piel artificial y equivalentes de piel de bioingeniería). Sin embargo, por ser un tratamiento en estudio y poco recurrente se encuentra con resultados en proceso por el momento, convirtiéndose en una nueva técnica y alternativa de tratamiento para las UV de difícil manejo ${ }^{29}$.

En países como México se encuentra una variedad de tratamientos para las UV, entre ellos está el polímero polisacárido con óxido de zinc, el cual ayuda en la reducción del tamaño de las UCV en aproximadamente un $63.1 \%$ en alrededor de ocho semanas ${ }^{21}$, este tipo de tratamiento es un coadyuvante con el vendaje y compresiones en los tratamientos tradicionales de las UV. Además, se encuentra la pentoxifilina, la cual ha demostrado efectividad en los tratamientos de UV como coadyuvante farmacológico en los apósitos y vendajes compresivos ${ }^{7}$, sin embargo este tipo de tratamientos no está indicado las UV clasificadas como CEAP (Clinical-Etiological-Anatomical-Pathophysiological) $\mathrm{C} 6$, incrementado costos de tratamiento con beneficios nulos generando pérdida importante en la economía del sistema de salud de México?.

\section{Discusión}

Las heridas crónicas en este caso las UV son de gran impacto económico para el sector de la salud, en los países occidentales los costos de estos tratamientos representan el $2-5 \%$ del presupuesto total de salud, además de la alta prevalencia de estas heridas ${ }^{12}$.

Nova, et al. (2017) ${ }^{17}$, en su artículo sobre la curación avanzada y la curación tradicional de las UV, en donde la curación tradicional es la compresión y desbridación de la herida por el tiempo que se determine, mientras que en la curación avanzada se realizan 20 procedimientos de 30 minutos de duración con el uso de apósitos activos, soluciones de limpieza, protectores cutáneos y sistemas de elastocompresión, logrando de esta manera que los costos se aumenten hasta cinco veces más. Los resultados de este estudio determinan que los costos de la curación avanzada durante cinco meses son mucho menos que los de los 48 meses de la curación tradicional, debido a que el número de curaciones de la tradicional a la avanzada tiene una diferencia de 12 curaciones por mes, en conclusión, este estudio evidencia en la curación tradicional un costo de USD 4,178 y en la curación avanzada un costo total de USD 691.

La terapia de compresión monocapa con apósito primario, en donde el costo de 21 agentes de curado es de USD 175.62 dólares y el cierre de la herida alcanzo un $97.1 \%$ es un tratamiento económico y con menor tiempo de cicatrización, siendo opción cuando los recursos financieros son escasos ${ }^{25}$. 
En Australia entre el 40 y $60 \%$ de los pacientes no recibieron la terapia adecuada de compresión, asociando esto con la falta de información para el paciente, los gastos para los pacientes mayores de 60 años se han estimado en más de USD 27.5 millones al año ${ }^{30}$.

En EE.UU. se utilizó el modelo analítico de decisión de Márkov para comparar el costo incremental y las semanas de curación de las UV entre los tratamientos de presión negativa de un solo uso y el tratamiento tradicional; donde se encontró un ahorro en costos de USD 7,756 por paciente y una reducción esperada de 1.67 semanas de úlceras abiertas durante 12 semanas y una reducción de costos de USD15,749 durante 26 semanas, siendo el tratamiento de presión negativa de un solo uso el más rentable ${ }^{20}$. Además se encontró el sistema de extracción de autoinjerto epidérmico para la curación de estas heridas, en donde el $62 \%$ de una muestra de 13 pacientes sanaron en cuatro meses, cuatro de ellos sanaron en un mes, al utilizar el autoinjerto reduce los costos de atención médica basados en un promedio de USD 1,153 por paciente y ahorro USD 650 al centro de heridas, al brindar la efectividad en el $63 \%$ de los pacientes mejora los costos poscicatrización de heridas y las finanzas del centro de heridas ${ }^{20}$. Al consultar las guías de práctica clínica de la sociedad de cirugía vascular y el foro venoso americano recomiendan que los injertos de piel y las terapias basadas en células representan una estrategia de segunda línea cuando falla un mínimo de cuatro a seis semanas de terapia estándar para heridas.

En Reino Unido el análisis de costo-efectividad de la ablación con láser costó USD 606.73 más que la esclerosis de espuma y la cirugía costó USD 381.19 más que la ablación con láser, en un umbral de pagar USD 26,379.64 dólares, teniendo en cuenta que se consideró superior a la cirugía en este estudio. Por otro lado, la comparación de escleroterapia con la cirugía costó USD 972.09 adicionales por paciente, lo que resulta en una relación costo-efectividad incremental de USD 23,153 dólares ${ }^{31}$.

Jiménez y Quiroga (2017) ${ }^{22}$, en relación con la escleroterapia afirman que este tratamiento no ha tenido los resultados esperados, de acuerdo con la tasa de reincidencia y complicaciones por migración del agente a la circulación sistémica, siendo recomendado en varices menores a $3 \mathrm{~mm}$ (telangiectasias), varices recurrentes o tratamiento de venas perforantes asociadas a ulceración.

Otra de las alternativas de tratamiento son los apósitos, convirtiéndose en opción teniendo en cuenta que las UV requieren tiempos prolongados de cuidado incrementando costos, existen varios tipos de apósitos que contienen agentes antimicrobianos que controlan la infección y ayudan en la cicatrización de heridas complejas tales como úlceras crónicas ${ }^{32}$.

Los apósitos de los pacientes se cambian inicialmente cada 2-3 días y los pacientes continúan durante 1-9 meses, además de ser importante el sistema de compresiones que se le va a realizar a cada uno de los pacientes ${ }^{32}$. Por esta razón se debe tener el conocimiento sobre la calidad del apósito que utilizar, el apósito apropiado se considera aquel que mantiene un medio húmedo, controla el exudado, tiene una adhesión a piel sin macerar tejido y de uso fácil, aún no se han logrado identificar características definitorias entre apósitos ${ }^{3}$.

Un estudio comparativo demostró la efectividad del uso de las membranas multifuncionales sobre el uso de apósitos húmedos o secos para heridas crónicas, confirmando de esta manera que los apósitos convencionales ocasionan en la mayoría de casos laceración en la zona afectada al momento de retirarlas. Al apreciar complicaciones como el dolor con frecuencia traumático y la alteración del lecho de la herida conlleva el aumento de los casos de incumplimiento y abandono del tratamiento, siendo la membrana multifuncional menos dolorosa ${ }^{33}$.

El tratamiento de apósitos en el hogar es un $62.42 \%$ menos costoso e incentiva a las personas el autocuidado, obteniendo resultados positivos no solo para la curación de la UV, sino disminución en los costos de salud y mejora de la calidad de vida ${ }^{14}$.

En relación con los injertos de piel y los estudiados que se adelantan en China, las guías de práctica clínica de la sociedad de cirugía vascular y el foro venoso americano recomiendan que los injertos de piel y las terapias basadas en células representan una estrategia de segunda línea cuando falla un mínimo de cuatro a seis semanas de terapia estándar para heridas.

Las limitaciones de esta revisión es el reducido número de estudios frente a los costos de los tratamientos de UV no solo en América Latina, sino en países de Europa y Asia. Es importante aumentar este tipo de estudios para abordar este problema de salud pública y mitigar el impacto a futuro.

\section{Conclusiones}

En el mundo existen diferentes abordajes y dispositivos para el tratamiento de UV y su aplicación depende de la disponibilidad; en la revisión se hallaron costos por tratamientos invasivos (quirúrgicos, escleroterapia, ablación por radiofrecuencia, injertos de piel) y no invasivos (coadyuvantes farmacológicos, apósitos 
de diversos compuestos, terapia de compresión y geles con diferentes principios activos), los cuales varían según la inversión de cada país.

\section{Financiamiento}

La presente investigación no ha recibido ninguna beca específica de agencias de los sectores públicos, comercial o con ánimo de lucro.

\section{Conflicto de intereses}

Los autores declaran no tener conflicto de intereses.

\section{Responsabilidades éticas}

Protección de personas y animales. Los autores declaran que para esta investigación no se han realizado experimentos en seres humanos ni en animales.

Confidencialidad de los datos. Los autores declaran que han seguido los protocolos de su centro de trabajo sobre la publicación de datos de pacientes.

Derecho a la privacidad y consentimiento informado. Los autores declaran que en este artículo no aparecen datos de pacientes.

\section{Bibliografía}

1. Salud de las piernas e insuficiencia venosa crónica [Internet]. Cinfa avalado por Capítulo Español de Flebología y Linfología; 2014. Disponible en: https://www.consejogeneralenfermeria.org/docs_revista/Dossier_ Salud_de_las_Piernas_e_IVC_Cinfa.pdf

2. Vázquez-Hernández I, Acevedo-Peña M. Prevalencia de insuficiencia venosa periférica en el personal de enfermería. Enfermería Univ. 2016;13(3):166-70.

3. Tafernaberry G, Otero G, Agorio C, Dapueto JJ. Adaptación y evaluación inicial del Charing Croos Venous Ulcer Questionnaire en pacientes con úlceras venosas crónicas en Uruguay. Rev Med Chile. 2016;44(1):55-65.

4. González Consuegra RV, Gómez Ochoa AM. Contexto social, biológico, psicológico, económico y cultural en personas con heridas en miembros inferiores. Avances en Enfermería. 2008:26(1):75-84.

5. Lopes Macêdo MM, Silva Souza DA, dos Santos JC, Nogueira Rodrigues $\mathrm{R}$, de Sousa Afonso G, de Oliveira Henriques Cortez A, et al. Úlcera venosa: seis años de existencia por 92 días de cicatrización. Gerokomos. 2016;27(3):131-3

6. Cedeño Quinapallo VA, Molina Chaca LB. Aplicación del proceso de atención de enfermería en pacientes con úlceras por presión en el Hospital Universitario, año 2015 [tesis en internet]. [Ecuador, Cantón Guayaquil]: Hospital Universitario de Guayaquil; 2015. Disponible en: http:// repositorio.ug.edu.ec/handle/redug/8170

7. Gohel MS, Heatley F, Liu X, Bradbury A, Bulbulia R, Cullum N, et al. A Randomized Trial of Early Endovenous Ablation in Venous Ulceration. NEJM. 2018;378(22):2105-2114

8. Sánchez-nicolat DNE, Guardado-bermúdez F, Arriaga-caballero JE, Torres-martínez JA, et al. Revisión en úlceras venosas: Epidemiología, fisiopatología, diagnóstico y tratamiento actual. Rev Mex Angiol. 2019;47(1):26-38.

9. Delgado Roche E, Malla Clua B, MArquilles Bonet C, Bravo Andrés N Abordaje de una úlcera de hidrodetersivo y vendaje de compresión multicapa. Gerokomos. 2017;28(4):208-11.
10. Quintero RSG, Ruiz RL, Legrá MN, Quintero Lores CM. Elementos caracteristicos de costos relacionados con la salud pública en Cuba. Revista Información Científica. 2017;96(4):786-97.

11. Chávez-Reyes G, Chirinos-Caraza SJ. Safenectomía ambulatoria segmentaria Ambulatory segmentary safenectomy. Acta Médica Peruana. 2006;23(1):56-8.

12. Noppeney $T$, Storck M, Nüllen $H$, Schmedt C-G, Kellersmann R, Böckler $D$, et al. Perioperative quality assessment of varicose vein surgery: Commission for quality assessment of the German Society for Vascular Surgery. Langenbecks Arco Sung. 2016;401:375-80.

13. Kheirelseid EA, Bashar K, Aherne T, Babiker T, Naughton P, Moneley $D$, et al. Evidence for varicose vein surgery in venous leg ulceration. The Surgeon. 2016;14(4):219-233.

14. Aherne T, McHugh SM, Tashkandi W, Byrne J, Aljabi Y, Moneley D, et al. Radiofrequency ablation: An assessment of clinical and cost efficacy. Ir. J. Med. Sci. 2016;185(1):107-110.

15. Rai A, Porsalman $M$, Khatony $A$, \& Sobhiyeh $M$. Comparison of foam sclerotherapy versus radiofrequency ablation in the treatment of primary varicose veins due to incompetent great saphenous vein: Randomized clinical trial. J. Vasc. Nurs. 2019;37(4):226-231.

16. Critello CD, Pullano SA, Matula TJ, De Franciscis S, Serra R, \& Fiorillo AS. Recent developments on foaming mechanical and electronic techniques for the management of varicose veins. Expert Rev. Med. Devices. 2019:16(11):931-940.

17. Rodríguez JM, Díaz PA, Hoyos DC \& Pinto JM. Impacto de costo efectividad en la cura avanzada versus cura tradicional en ulcera venosa. Enferm Dermatol. 2017;11(32):40-44.

18. Guest JF, Rana K, Singh H, \& Vowden P. Cost-effectiveness of using a collagen-containing dressing plus compression therapy in non-healing venous leg ulcers. J. Wound Care. 2018;27(2):68-78.

19. Guest JF, Fuller GW, \& Vowden P. Venous leg ulcer management in clinical practice in the UK: Costs and outcomes. Int. Wound J. 2018;15(1):29-37.

20. Januário V, Ávila DA, Penetra MA, Sampaio AL, Noronha MI, Cassia FD, et al. Evaluation of treatment with carboxymethylcellulose on chronic venous ulcers. An Bras Dermatol. 2016;91(1):17-22.

21. Moreno-Eutimio MA, Moreno J, Cueto-García J. Efecto de un polímero polisacárido con óxido de zinc en la reducción del tamaño de las úlceras venosas crónicas. Rev Mex Angiol. 2016;44(2):67-71.

22. Jiménez CE, Quiroga F. Radiofrecuencia en el tratamiento de las varices de los miembros inferiores. Estudio prospecivo en 176 pacientes en Bogotá. Angiología. 2017;69(2):1-9

23. Oliveira AP De, Guitton B, Baptista R. Cost of the treatment for venous ulcers in an outpatient clinic and in the household: A descriptive study. Brazilian J Nurs. 2015;14:221-8.

24. Walzer S, Dröschel D, Vollmer L, Atkin L, \& Ousey, K. A cost-effectiveness analysis of a hydration response technology dressing in the treatment of venous leg ulcers in the UK. J. Wound Care. 2018;27(3):166172.

25. Augustin M, Herberger K, Kroeger K, Muenter KC, Goepel L \& Rychlik R. Cost-effectiveness of treating vascular leg ulcers with UrgoStart $₫$ and UrgoCell囚. Int. Wound J. 2016;13(1):82-87.

26. Sabolinski ML, Gibbons G. Comparative effectiveness of a bilayered living cellular construct and an acellular fetal bovine collagen dressing in the treatment of venous leg ulcers. J Comp Eff Res. 2018;7(8):797805.

27. Atkin L, Schofield A, Kilroy-Findley A. Updated leg ulcer pathway: Improving healing times and reducing costs. Br J Nurs. 2019;28(20):S21-S26

28. Toledo RR, de Castro Santos ME, \& Schnaider TB. Effect of pycnogeno on the healing of venous ulcers. Ann Vasc Surg. 2017;38:212-219.

29. Pan J, Hu X, Yin H, Zhang C, Yan Z. Effectiveness of different types of skin grafting for treating venous leg ulcers. Medicine (Baltimore). 2021:15:13-6.

30. Cheng Q, Gibb M, Graves N, Finlayson K, Pacella RE. Cost-effectiveness analysis of guideline-based optimal care for venous leg ulcers in Australia. BMC Heal Serv Res. 2018;18(421):1-13

31. Brittenden J, Cooper D, Dimitrova M, Scotland G, Cotton SC, Elders A et al. Five-year outcomes of a randomized trial of treatments for varicose veins. N Engl J Med. 2019;381:912-22.

32. Hämmerle G, Strohal R. Efficacy, and cost-effectiveness of octenidine wound gel in the treatment of chronic venous leg ulcers in comparison to modern wound dressings. Int Wound J. 2016;13(2):182-8.

33. Zapata Dioses RE, Condori Quijandria MC. Efectividad de las diferentes curas avanzadas en la disminucion de las úlceras y heridas crónicas [tesis en internet]. [Lima, Perú]: Universidad Norbert Wiener, Facultad de Ciencias de la Salud; 2019. Disponible en: http://repositorio.uwiener.edu. pe/xmlui/handle/123456789/3183 